\title{
IFN Regulatory Factor-1 Modulates the Function of Dendritic Cells in Patients with Acute Coronary Syndrome
}

\author{
Min Guo Rui Yan ${ }^{b}$ Caixia Wang ${ }^{a}$ Hongtao Shia Meng Sun ${ }^{a}$ Shuang Guo \\ Chuanshi Xiao
}

aDepartment of Cardiology, The First Hospital of Shanxi Medical University, Taiyuan, China, ${ }^{b}$ Nursing College of Shanxi Medical University, Taiyuan, China

\section{Key Words}

Atherosclerosis • Acute coronary syndrome $・$ IRF-1 $・$ Dendritic cells $・$ Inflammation

\begin{abstract}
Background: Atherosclerosis is widely recognized as a complex inflammatory disease involving pathogenic immune response of T cells and antigen-presenting cells (APCs) such as dendritic cells (DCs) and macrophages. Accumulating evidence has revealed that mature DCs play critical roles in the differentiation of effector T cells into CD4+ T cells, which effectively participate in the onset of acute coronary syndrome (ACS). IFN regulatory factor (IRF)- 1 has been shown to be involved in various immune processes. The role of IRF-1 in DCs in the pathogenesis of ACS has not been investigated. Methods and Results: We examined the relative mRNA and protein expression of IRF-1 in human monocyte-derived DCs in patients with coronary artery disease (CAD). The overexpression or silencing of IRF-1 expression in DCs in patients with ACS was performed to explore the possible role of IRF- 1 in the maturation and function of DCs involved in ACS. The results showed that the relative expression of IRF-1 in DCs is obviously increased in patients with ACS. The overexpression or silencing of IRF-1 expression could effectively promote or attenuate the maturation and function of DCs. In addition, we revealed that the MAPK pathway (phosphorylation of JNK, p38 and ERK1/2) might be downstream of IRF-1 signalling pathway in activation of circulating DCs in ACS patients. Conclusion: The present data demonstrate that IRF-1 could effectively promote the immune maturation and function of DCs in ACS patients.

M. Guo and R. Yan contributed to the work equally.

Chuanshi Xiao

KARGER 125
Department of Cardiology, The First Hospital of Shanxi Medical University Taiyuan, 030001 (China)

Tel. +86-351-4639551, Fax +86-351-4639551, E-Mail 43375407@qq.com 


\section{Cellular Physiology Cell Physiol Biochem 2015;36:599-610 \begin{tabular}{l|l} 
DOI: 10.1159/000430123 & (C) 2015 S. Karger AG, Basel
\end{tabular} \begin{tabular}{l|l|l|l|l} 
and Biochemistry Published online: May 18, 2015 & www.karger.com/cpb \\
\hline
\end{tabular} \\ Guo et al.: IRF-1 Modulates the Function of DCs in ACS Patients}

\section{Introduction}

Atherosclerosis (AS) has been recognized as a chronic inflammatory disease involving both innate and adaptive immunity that occurs within the artery wall [1-3]. Its clinical consequence, coronary artery disease (CAD), is the leading cause of morbidity and mortality worldwide [4,5]. Dendritic cells (DCs) are professional antigen-presenting cells that specialize in capturing, processing and presenting antigens (viral, bacterial, oxidized LDL (ox-LDL) and HSP et al) and it has a critical role in naïve T cells differentiation to effector $\mathrm{T}$ cells to induce and control immunity [6-8]. Studies have shown that DCs can be found in the normal mouse aortic intima in areas predisposed to developing atherosclerotic lesions and are rarely found in areas protected from arthrosclerosis $[9,10]$. DCs have also been found in atherosclerotic lesions in human and mouse models [11,12]. In recent years, accumulating evidence indicates that DCs and T cells are present in clusters, localize to the shoulder region and the ruptured areas of the lesions and play important roles in the initiation, progression and rupture of atherosclerotic lesions, eventually leading to acute coronary syndrome (ACS), including unstable angina (UA) and acute myocardial infarction (AMI) [13-15]. It has been demonstrated that DCs are closely associated with the pathogenesis of coronary artery disease. Ranjit et al found that DCs in patients with ACS were activated [16] and TLR4 and MAPK families have been shown to be involved in the activation of circulating DCs in ACS patients [17]. However hitherto, the exact role and mechanisms of DCs in the pathogenesis of atherosclerosis and ACS have remained unclear.

Transcription factors of the IFN regulatory factor family were initially identified as regulators of interferon- $\alpha / \beta$ genes. These factors efficiently participate in many innate and adaptive immune responses. Several studies indicate that the family members such as IRF-8, IRF-2 and IRF-4 play important roles in the development and maturation of DCs [18]. IRF-1 was first identified as an IRF family member by its ability to participate in immunomodulation and hematopoietic differentiation [19-21]. Extensive analysis of IRF-1 revealed that it plays essential roles in the development and function of specific cell populations of the immune system [19]. IRF-1-/- mice exhibit impaired NK cell maturation, reduced macrophage IL12 production and defective Th1 responses [21-23]. These mice are highly susceptible to Leishmania mania and Lymphocytic choriomeningitis viral infections which are closely associated with Th1 responses in normal mice [23]. In contrast, these mice exhibit reduced susceptibility to experimental autoimmune encephalomyelitis and diabetes [24-26]. Our previous study has shown that IRF-1 siRNA down-regulates Th1 cell function in patients with ACS [27]. Evidence has also emerged that IRF-1-/- mice exhibit a predominance of DC subsets with an immature and tolerogenic phenotype and reduced numbers of conventional DCs [28]. Given the potential role of IRF-1 in regulating inflammation and autoimmunity, we hypothesized that IRF-1 plays causative roles in the pathogenesis of ACS, which is processes finely modulated by DCs.

In the present study, we sought to elucidate the possible transcriptional mechanism underlying the activation of the plaque destabilization and the onset of ACS. We found that the expression of IRF-1 was upregulated in human monocyte-derived DCs in patients with ACS. The Overexpression or silencing of IRF-1 expression could effectively promote or attenuate the maturation of DCs. Addtionally, we explored the exact role of IRF-1 in the function of DCs and found that IRF-1 obviously modulated the uptake of ox-LDL as well as the secretion of pro-inflammatory cytokines by DCs. Furthermore, in this study, we sought to identify the possible mechanism IRF-1 might modulate the maturation of DCs by inducing the phosphorylation of MAPK family (including ERK, JNK and p38) in DCs from ACS patients. These findings highlight a unique role for IRF-1 in DC activity and indicate IRF-1 is a novel target in the progression and deterioration of ACS. 


\section{Cellular Physiology Cell Physiol Biochem 2015;36:599-610 \begin{tabular}{l|l|l}
\hline DOI: 10.1159/000430123 & (C)15 S. Karger AG, Basel
\end{tabular} and Biochemistry Published online: May 18, $2015 \quad$ www.karger.com/cpb

\section{Materials and Methods}

Patients and control subjects

The study protocol conforms to the ethical guidelines of the 1975 Declaration of Helsinki and was approved by the Ethics Committee of the First Hospital of Shanxi Medical University. Informed consent was obtained from all cases. 105 consecutive patients with angiographically verified CAD were selected from the cardiology department in the hospital from February to October. None of the patients had a history of thromboembolism, renal failure, malignant tumor, advanced liver disease, other inflammatory disease, dilated cardiomyopathy or valvular heart disease. Four groups of subjects were studied.

Group I : The CPS group included 17 men and 6 women, inclusion criteria: clinical presentation of chest pain without any electrocardiographic changes, coronary artery stenosis or coronary spasm when acetylcholine was given through intracoronary injection during arteriography.

Group II : The SA group included 18 men and 7 women, inclusion criteria: clinical presentation of discomfort in the chest, jaw, shoulder, back or arm with downsloping or horizontal ST-segment depression $>1$ $\mathrm{mm}$ in an exercise test and can be relieved with nitroglycerin or rest.

Group III: The UA group included 23 men and 7 women, inclusion criteria: angina pectoris with an accelerating pattern, or prolonged duration $(>20 \mathrm{~min})$, or recurrent episodes at rest or within minimal effort, with ischemic electrocardiographic changes such as ST-segment changes and/or T-wave inversion.

Group IV: The AMI group included 22 men and 5 women, inclusion criteria: chest pain lasting $>30 \mathrm{~min}$ within the $24 \mathrm{~h}$ before enrollment, that was not relieved by nitroglycerin, and muscular injury evidence by significantly rise of creatine kinase-MB and troponin I levels.

\section{Blood samples and cell culture}

Blood samples were drawn from all of the patients using 21-gauge needles for clean venipuncture of an antecubital vein on the morning after arrival. The time between the onset of chest pain and the performance of phlebotomy was $<24 \mathrm{~h}$. Blood was drawn into sodium heparin vacutainers (Becton Dickinson, Cedex, France) and then centrifuged for $15 \mathrm{~min}$ at $1000 \times \mathrm{g}$. Peripheral blood monocyte cells (PBMCs) were isolated from peripheral blood samples by density gradient centrifugation (2000 rounds/min for $20 \mathrm{~min}$ ) with Ficoll-hypaque (Sigma Chemical Co., St. Louis, MO) to remove red blood and granulocytes. Monocytes were collected from the PBMCs using a MACS column containing Abs conjugated to CD14 (Miltenyi Biotec, Bergisch Gladbach, Germany). Cell purity was confirmed by flow cytometry (90-95\%). Monocytes $\left(1 \times 10^{6}\right)$ were then plated in six-well culture plates in 3ml DC culture medium (RPMI-1640 medium, $10 \%$ fetal bovine serum, $2 \mathrm{mM}$ L-glutamine, $100 \mathrm{U} / \mathrm{ml}$ streptomycin/penicillin and $2.5 \mu \mathrm{g} / \mathrm{ml}$ Plasmocin) supplemented with $100 \mathrm{ng} / \mathrm{ml} \mathrm{recombinant} \mathrm{human} \mathrm{granulocyte} \mathrm{-macrophage} \mathrm{colony-stimulating} \mathrm{factor} \mathrm{(rh} \mathrm{GM-CSF)} \mathrm{(PeproTech}$ EC, London, UK) and 100ng/ml recombinant human IL-4 (rh IL-4) (PeproTech EC, London, UK) and were cultured for 6 days in a humidified atmosphere of $5 \% \mathrm{CO}_{2}$ maintained at $37^{\circ} \mathrm{C}$. After 6 days, the cultures developed clusters of DC colonies. The percentage of DC viability was measured by a Trypan blue exclusion assay (90-95\%).

\section{Synthesis of IRF-1 siRNA}

The siRNA sequences used for the targeted silencing of IRF-1 were obtained from Invitrogen (Invitrogen, Carlsbad, CA), as previously described [27]. The IRF-1 siRNA sequences were as follows: sense 5'-AAGTAATTTCCCTTCCTCATCTATAGTGAGTCGTATTAGGATCC-3' and antisense 5'-AAGATGAGGAAGGGAAATTACTATAGTGAGTCGTATTAGGATCC-3'.

\section{Lentiviral vector construction}

The lentiviral vector pGC-FU-IRF-1 (GenBank accession No. NM_012591.1) was constructed (GeneChem, Shanghai, China). The viral particles were generated by the cotransfection of 293T cells with the pGC-FU-IRF-1 plasmid and two packaging vectors (pHelper1.0 and pHelper2.0 plasmids) using Lipofectamine2000 (Invitrogen, Carlsbad, CA). After 48 hours, supernatants were collected from these cells, passed through a $0.45 \mu \mathrm{m}$ filter, aliquoted, and stored at $-80^{\circ} \mathrm{C}$. The lentivirus carried EGFP, therefore, the viral titer was determined by counting the percentage of GFP-positive cells at $\times 100$ magnification under a fluorescent microscope (Olympus, Tokyo, Japan).

\section{KARGER}




\section{Cellular Physiology Cell Physiol Biochem 2015;36:599-610 \begin{tabular}{l|l|l}
\hline DOI: 10.1159/000430123 & O 2015 S. Karger AG, Base
\end{tabular} www.karger.com/cpb \\ Guo et al.: IRF-1 Modulates the Function of DCs in ACS Patients}

Cell isolation and gene transfection in vitro

One day before transfection, DCs from patients with ACS were cultured in Opti-OMEM without serum and antibiotics. The cells $\left(5 \times 10^{5}\right)$ were aliquoted into twelve-well culture plates and classified into three groups: ACS group; IRF-1 siRNA group (transfected with 50nM IRF-1 using Lipofectamine2000) and IRF-1 group (transfected with $3 \mu \mathrm{g}$ pGC-FU-IRF-1 using Lipofectamine2000). Twelve hours after transfection, we decanted the supernatants and added new RPIM-1640 medium with antibiotics.

Real-time quantitative reverse transcription PCR

GADPH, IRF-1, SR-A, CD36 and LOX-1 mRNA expression were analyzed by real-time quantitative reverse transcription PCR. Total cellular RNA from the human monocyte-derived DCs or transfected DCs was extracted using Trizol RNA extraction kit (Invitrogen, Carlsbad, CA, USA) according to the manufacturer's recommendations. The RNA concentration and purity were measured by a spectrophotometer. $5 \mu \mathrm{g}$ of total RNA was reversely transcribed into cDNA using a first-strand real-time polymerase chain reaction kit (TOYOBO, Osaka, Japan). $5 \mu \mathrm{l}$ cDNA was then used as a template for SYBR Green real-time PCR reactions (TaKaRa, Kyoto, Japan). The PCR reaction mixture was prepared with PCR buffer, SYBR Green Taq DNA polymerase and the specific primers for GADPH, IRF-1, SR-A, CD36 and LOX-1 (Table 1) following the manufacturer's protocol. The mRNA levels were quantified by the Relative Quantification Study using ABI7500 system SDS software (Applied Biosystems, Foster City, CA), and the copies of each mRNA were determined by the standard curve method.

\section{Western blot analysis}

Total proteins were extracted from control DCs and transfected DCs. The protein concentrations were assessed by BCA kits (Piece, Carlsbad, CA, USA) according to the manufacturer's recommendations. 50 $\mathrm{gg}$ protein were heated to $99^{\circ} \mathrm{C}$ for $10 \mathrm{~min}$ and then subjected to $9 \%$ sodium dodecyl sulfate-polyacrylamide gel electrophoresis (SDS-PAGE) and then transferred to nitrocellulose membranes. Membranes were blocked with blocking buffer ( $5 \%$ non-fat milk in TBS containing $0.3 \%$ Tween-20) for 2 hours, washed and then incubated overnight at $4^{\circ} \mathrm{C}$ in a 1:2000 dilution of specific antibodies against the IRF-1, SR-A, CD36, LOX-1, JNK, P38 and ERK (all from Abcam, Cambridge, UK). After three washes, horseradish peroxidase-conjugated secondary antibodies were added for $2 \mathrm{~h}$ at room temperature. Finally, membranes were washed three times, and the signal was detected by using ECL according to the manufacturer's protocol (PIERCE, Rockford, IL). Protein levels were quantified by scanning the bands intensities by NIH Image Version 1.61.

\section{Identifying the maturation state of DCs}

DCs from different groups were aliquoted into tubes and washed twice with phosphate-buffered saline (PBS). DC phenotypes were analyzed on a flow cytometer (FACS Calibur, Becton Dickinson) using antibodies against CD83-FITC, CD86-FITC and the corresponding isotype controls. Briefly, $20 \mu \mathrm{l}$ antibody was incubated with $100 \mu \mathrm{l}$ cell suspension for $20 \mathrm{~min}$ at room temperature respectively. Then, the mixture was washed in staining buffer containing $2 \%$ bovine serum albumin and $0.1 \%$ NaN3. The cells were resuspended in $500 \mu l$ buffer and immediately were analyzed by flow cytometry (FACS Calibur, Becton Dickinson).

Uptake of oxidized LDL (ox- $L D L)$

To measure the uptake and accumulation of Dil-ox-LDL, the transfected DCs were aliquoted into twelvewell culture plates in RPMI-1640 medium containing 0.1\% FBS for $24 \mathrm{~h}$ and then incubated with $20 \mu \mathrm{g} / \mathrm{ml}$ Dil-ox-LDL (Biomedical Technologies, Inc.) for an additional $4 \mathrm{~h}$ at $37^{\circ} \mathrm{C}$. DCs were washed with cold PBS and fixed with $2 \%$ formaldehyde. The Dil-ox-LDL incorporated fraction was analyzed by flow cytometry (FACS Calibur, Becton Dickinson). 


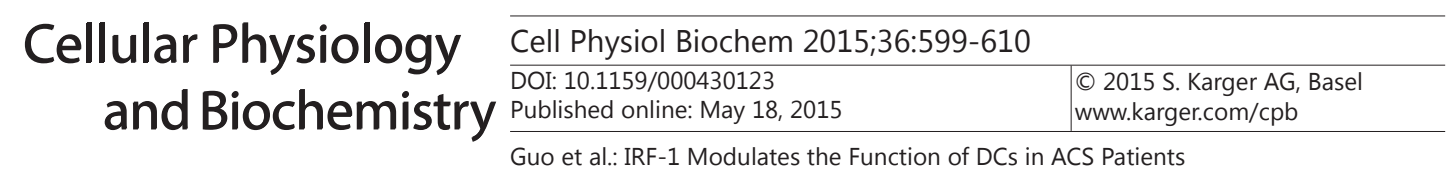

Table 2. Clinical characteristics of enrolled population

\begin{tabular}{lllll}
\hline characteristics & CPS(n=23) & SA(n=25) & UA(n=30) & AMI(n=27) \\
\hline Age(years) & $57.4 \pm 8.2$ & $59.8 \pm 7.6$ & $55.7 \pm 7.0$ & $61.3 \pm 6.9$ \\
Male/female & $17 / 6$ & $18 / 7$ & $23 / 7$ & $22 / 5$ \\
Risk factors & & & & \\
Hypertension & $8(34.8 \%)$ & $10(40 \%)$ & $11(36.7 \%)$ & $10(37 \%)$ \\
Diabetes & $6(26.1 \%)$ & $8(32 \%)$ & $10(33.3 \%)$ & $8(29.6 \%)$ \\
Hypercholesterolemia & $10(43.5 \%)$ & $11(44 \%)$ & $14(46.7 \%)$ & $12(44.4 \%)$ \\
Current smoker & $9(39.1 \%)$ & $11(44 \%)$ & $12(40 \%)$ & $12(44.4 \%)$ \\
Medication & & & & \\
Aspirin & $20(86.9 \%)$ & $22(88 \%)$ & $28(93 \%)$ & $24(88.9 \%)$ \\
$\beta$-blockers & $12(52.2 \%)$ & $16(64 \%)$ & $19(63.3 \%)$ & $16(59.2 \%)$ \\
Statins & $13(56.5 \%)$ & $16(64 \%)$ & $20(66.7 \%)$ & $19(70.4 \%)$ \\
ACEI or ARBs & $10(43.5 \%)$ & $11(44 \%)$ & $12(40 \%)$ & $11(40.7 \%)$ \\
Nitrates & $10(43.5 \%)$ & $10(40 \%)$ & $14(46.7 \%)$ & $11(40.7 \%)$ \\
\hline
\end{tabular}

DC cytokine detection

The pro-inflammatory cytokines (IL-6, IL-10, IL-12p70 and TNF- $\alpha$ ) in the supernatants of the transfected cells cultured in RPMI-1640 containing Dil-ox-LDL $(20 \mu \mathrm{g} / \mathrm{ml})$ were analyzed using enzymelinked immunosorbent assay (ELISA) kits (all from Bender MedSystems, Burlingame, CA) according to the manufacturer's protocols. Each sample was tested in triplicate.

Statistical analysis

All statistical analyses were performed with SPSS 15.0. The results are expressed as the mean \pm SD of at least three independent experiments. Signigicant differences were assessed by ANOVA and the NewmanKeuls test. P values of $<0.05$ were considered statistically significant.

\section{Results}

Clinical characteristics of the patients

The basic clinical characteristics of the patients are showed in Table 2. There were no significant differences in age, gender, risk factors and medications among the patients with CPS, SA, UA and AMI.

The expression of IRF-1 is higher in ACS patients

We first examined the relative mRNA and protein exression levels of IRF-1 in the monocyte-derived DCs of patients with CPS, SA, UA and AMI. The results showed that the relative expression of IRF-1 was significantly increased in patients with AMI and UA compared with patients with SA and CPS $\left({ }^{*} \mathrm{p}<0.01\right)$, whereas the difference between the CPS and SA groups was not significant (\#p>0.05) (Fig. 1).

IRF-1 induces the maturation of DCs

DCs from patients with ACS were transfected with IRF-1 siRNA and pGC-FU-IRF-1, and then the protein levels of CD83 and CD86 which are often used to assess the maturation status of DCs, were quantified by flow cytometry. As shown in Fig. 2, the frequencies of CD83+ and CD86+ DCs were significantly upregulated in the IRF-1 group compared with the ACS group $(p<0.01)$. In contrast, the frequencies of CD83+ and CD86+ DCs were obviously decreased in the IRF-1 siRNA group ( $\mathrm{p}<0.01)$. Based on these data, we consider that IRF-1 might effectively induce the maturation of monocyte-derived DCs and promote the inflammatory response in atherosclerosis and ACS. 
Fig. 1. The expression of IRF-1 in human monocyte derived DCs. a. The relative mRNA expression of IRF-1 was assayed by real-time PCR. b. The relative protein expression of IRF-1 was assayed by western-blot. The mRNA and protein expression both higher in UA and AMI patients compared with CPS and SA patients. ${ }^{*} \mathrm{p}<0.01$ compared with CPS and SA group. \#p>0.05 compared with CPS group.
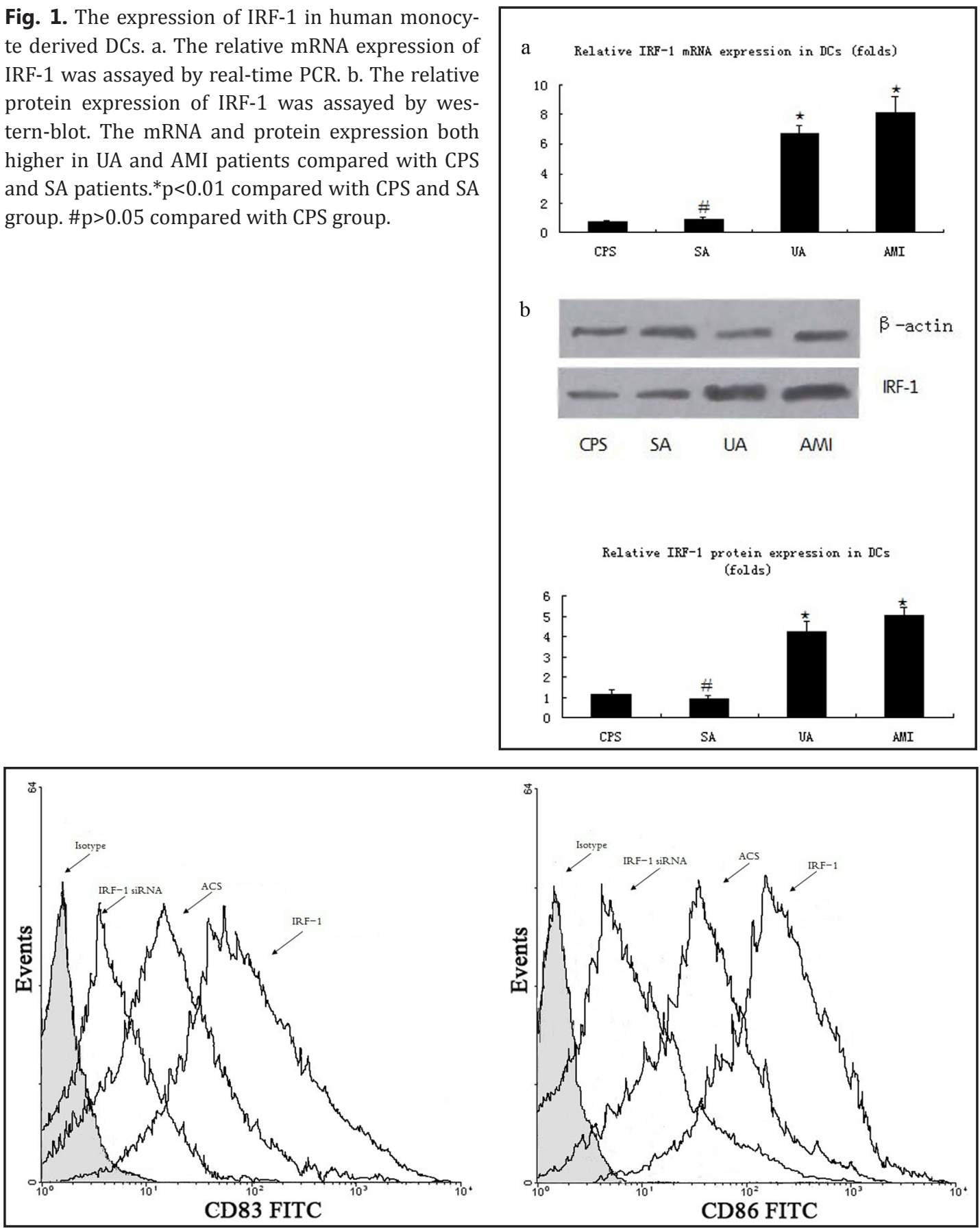

Fig. 2. FACS scan of CD83 and CD86 expression. The frequencies of CD83+ and CD86+ DCs were upregulated in IRF-1 group than in ACS group ( $p<0.01$ ). In contrast, The frequencies of CD83 and CD86 DCs were obviously decreased in IRF-1 siRNA group $(\mathrm{p}<0.01)$.

\section{IRF-1 promotes ox-LDL uptake by DCs}

There is evidence indicating that DCs comprise a large proportion of intimal foam cells and precede the appearance of macrophage-derived foam cells. We analyzed whether IRF-1 might induce the Dil-ox-LDL uptake by DCs in patients with ACS. The results were quantified by flow cytometry and showed that IRF-1 increased the ox-LDL-uptake capacity of DCs (Fig. $3)$.

\section{KARGER}


Fig. 3. Effects of IRF-1 on the uptake of Dil-oxLDL by DCs. The frequencies of Dil-oxLDL were upregulated in IRF1 group than in ACS group ( $\mathrm{p}<0.01)$. In contrast, the frequencies of Dil-oxLDL were obviously decreased in IRF-1siRNA group $(\mathrm{p}<0.01)$.
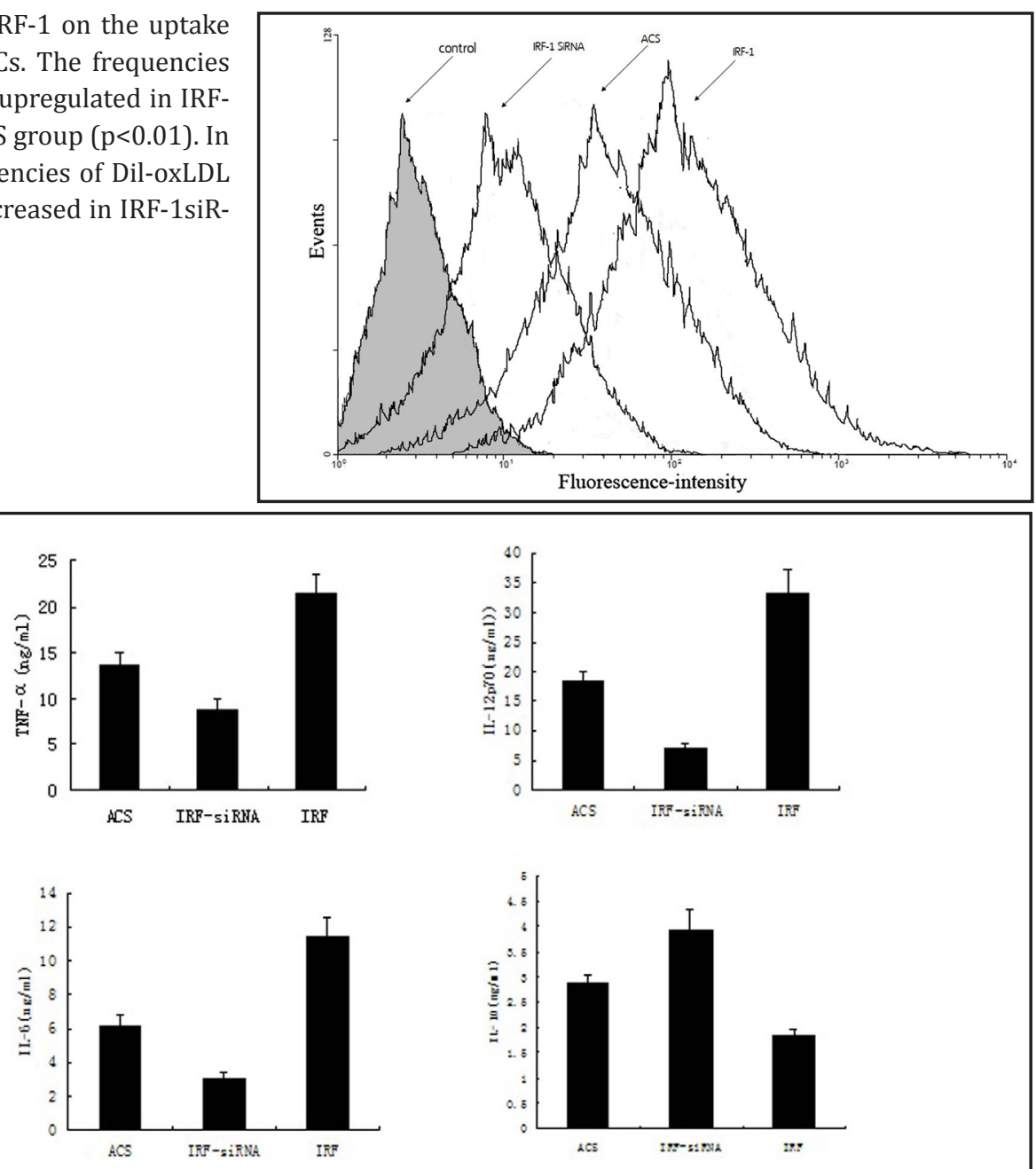

Fig. 4. The secretion of cytokines were assayed by ELISA. The IL-6, IL-12p70 and TNF- $\alpha$ concentration in the supernatant of transfected DCs were significant increased in IRF-1 group compared with ACS group $(\mathrm{p}<0.01)$, while the release of IL-10 is reduced compared with ACS group $(\mathrm{p}<0.01)$. Meawhile, the secretion of pro-inflammatory cytokines were decreased in IRF-1 siRNA group $(\mathrm{p}<0.01)$ and the secretion of IL-10 was increased in IRF-1 siRNA group $(\mathrm{p}<0.01)$.

IRF-1 modulates the secretion inflammation cytokines by DCs

Our results showed that the IL-6, IL-12p70 and TNF- $\alpha$ concentrations in the supernatants of transfected DCs stimulated with ox-LDL stimulation were significantly increased in the IRF-1 group compared with the ACS group $\left({ }^{*} p<0.01\right)$. In contrast, IL-10 concentrations in the supernatants of transfected DCs stimulated with ox-LDL were significantly decreased in the IRF-1 group compared with the ACS group ( $\left.{ }^{*} \mathrm{p}<0.01\right)$. Additionally, IL-6, IL-12p70 and TNF- $\alpha$ secretion was obviously decreased in the IRF-1 siRNA group ( ${ }^{*} p<0.01$ ) (Fig. 4). IL-10 concentrations in the supernatants of transfected DCs stimulated with ox-LDL were increased in the IRF-1 group compared with the ACS group $\left({ }^{*} \mathrm{p}<0.01\right)$.

\section{Effects of IRF-1 on SR-A, CD36 and LOX-1 expression in DCs}

Because scavenger receptor A (SR-A), CD36 and LOX-1 are well-known high-affinity scavenger receptors for ox-LDL, we examined the relative mRNA and protein levels of these genes. As shown in Fig. 5, our study showed that IRF-1 over-expression enhanced LOX-1 expression both at the mRNA and protein levels $\left({ }^{*} \mathrm{p}<0.01\right)$ but had no apparent effect on SR-A or CD36 expression (\#p>0.05). Conversely, IRF-1 siRNA could obviously reduce the expression of LOX-1 $\left({ }^{*} \mathrm{p}<0.01\right)$ but had no effect on the expression of SR-A or CD36 (\#p>0.05). 
Fig. 5. Relative mRNA and protein expression of scavenger receptors (SR-A, CD36 and LOX-1) in transfected DCs. The expression of LOX-1 significantly increased in IRF-1 group both at the mRNA and protein levels compared with ACS group and IRF-1 siRNA group $\left({ }^{*} p<0.01\right)$ but the expression of SR-A and CD36 showed no significantly difference in these groups (\#p>0.05). Conversely, the expression of LOX-1 obviously reduced in IRF-1 siRNA group $\left({ }^{*} \mathrm{p}<0.01\right)$ and also the expression of SR-A and CD36 showed no significantly difference in these three groups (\#p>0.05).

Assessment of the expression of the MAPK family proteins

Evidence has emerged that the immune state of DC is modulated through an exhaustive series of intracellular signaling pathways [29]. The highly conserved MAPK family is a major family that regulates cells by transducing extracellular signals into cellular responses [30, 31]. In this study, we found that the relative protein expression of phosphorylated MAPK family proteins, including JNK, p38 and ERK, was much higher in the IRF-1 group than the ACS group $\left({ }^{*} \mathrm{p}<0.01\right)$. In contrast, the relative protein expression of phosphorylated MAPK family proteins was significantly decreased in the IRF-1 siRNA group compared with the ACS group $(* \mathrm{p}<0.01)$ (Fig. 6).

\section{Discussion}

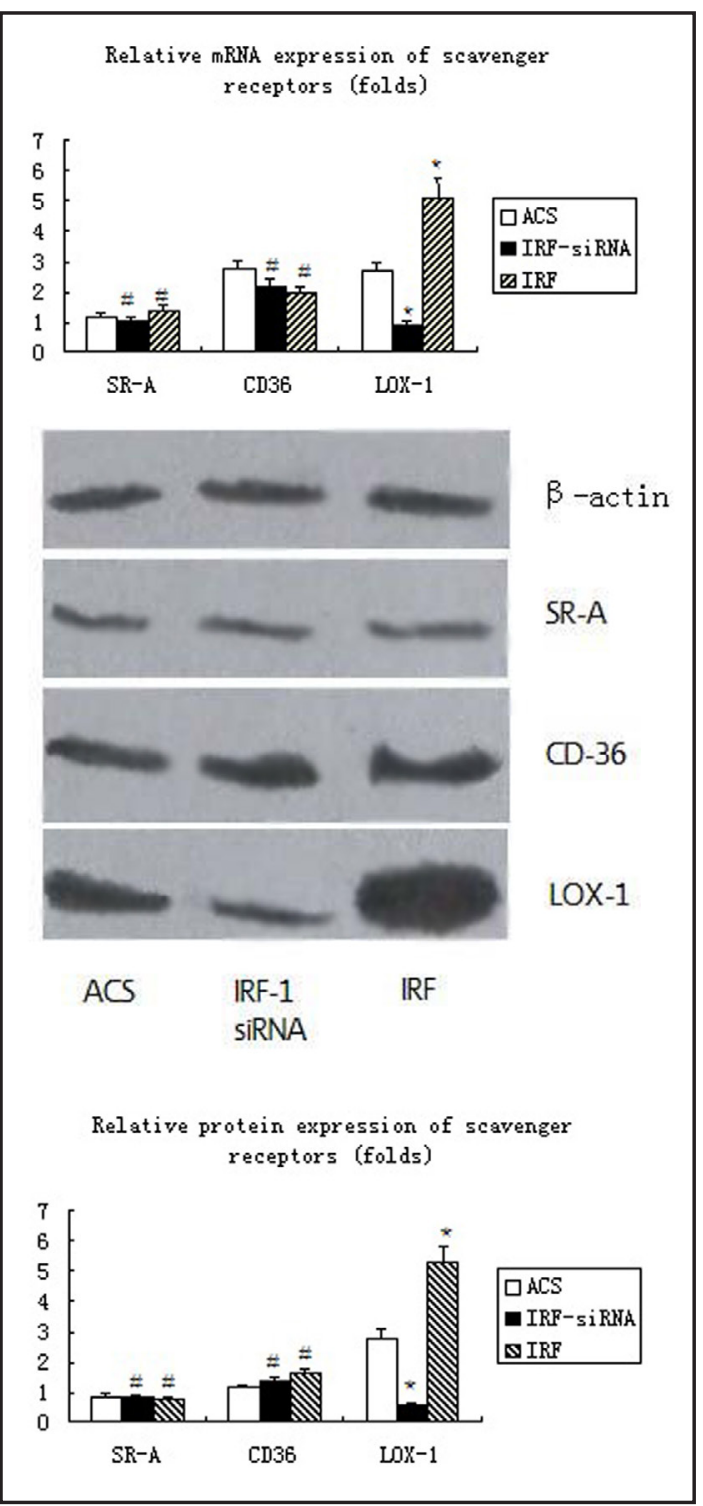

We had previously found that IRF-1 could participate the onset and progression of ACS by modulating the function of Th1 cells [27], but the function of IRF-1 in ACS still had not been elucidated. In the present study, we provided evidence for a novel role of IRF-1 in the pathogenesis of ACS. Our results demonstrated that the expression of IRF-1 in monocytederived DCs was obviously higher in patients with ACS both at the mRNA and protein levels. We found that IRF-1 upregulated the expression of co-stimulatory molecules such as CD83 and CD86 and activated the maturation of DCs. Furthermore, IRF-1 could potentially induce ox-LDL uptake and inflammatory cytokines secretion by monocyte-derived DCs in vitro. These findings indicated that IRF-1 may directly influence the activation of DCs and be involved in the onset of ACS.

Atherosclerosis, the major cause of CAD, is a multifactorial disorder in which immune mechanisms play critical roles. The activation of inflammation, which involves several types of immune cells and their effector chemokines and cytokines, leads to plaque destabilization and disruption as well as thrombus formation and eventually results in ACS [32-34]. As a critical player in innate and adaptive immune response, DCs have important roles in immune processes involved in atherosclerosis and in the initiation of ACS [17, 34, 35]. IRF- 
Fig. 6. Relative protein expression of MAPK family (JNK, P38 and ERK) in transfected DCs. The relative protein expression of phosphor-MAPK family was much greater expressed in IRF-1 group than ACS group $\left({ }^{*} \mathrm{p}<0.01\right)$. Oppositely, the relative protein expression of phosphor-MAPK family was significantly decreased in IRF-1 siRNA compared with ACS group $(* \mathrm{p}<0.01)$.

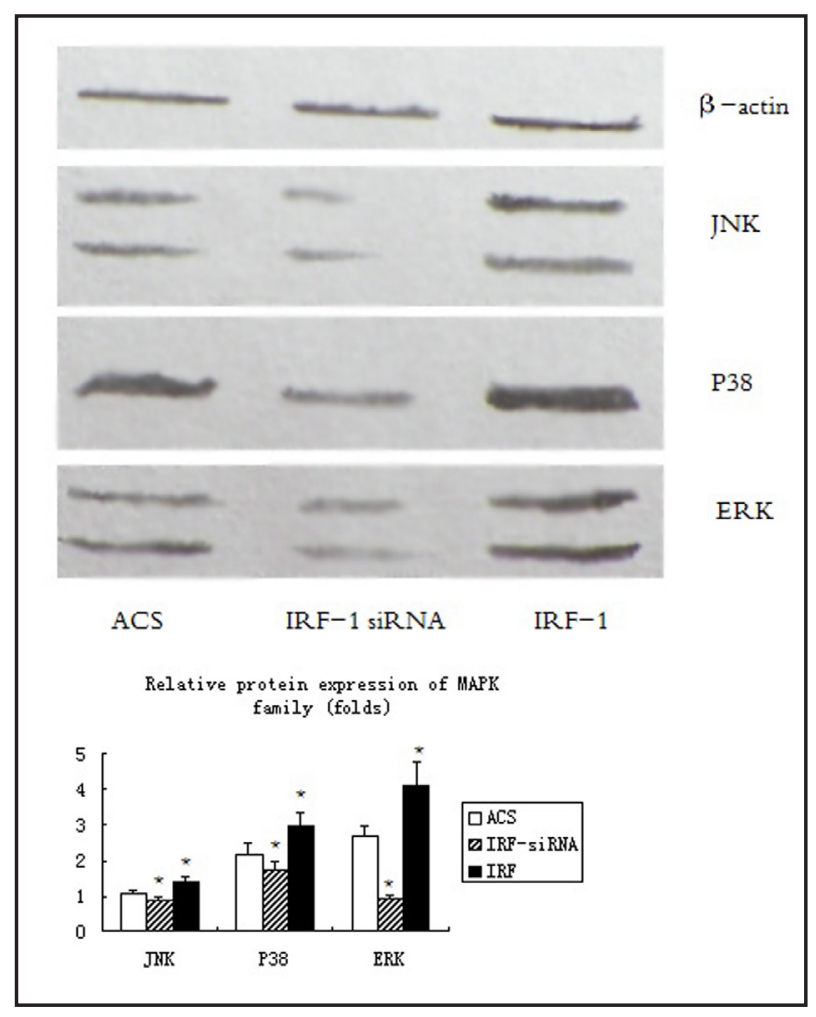

1 is a transcriptional factor that binds to specific DNA sequences of genes and controls the transcription of genes involved in mediating antiviral, immunomodulatory, anti-proliferative effects and apoptosis [36-38]. Evidence has indicated that IRF-1 participates in the process of atherosclerosis. It has been shown that the expression of IRF-1 is higher in human vascular lesions and that IRF-1 leads to growth inhibition in coronary smooth muscle cells [39]. Other findings have suggested that IRF-1 could mediate apoptosis in many cell types involved in the process of atherosclerosis [40-42]. A recent study found that DCs in IRF-1-/- mice exhibit an immature and tolerogenic phenotype and are unable to activate even following treatment with strong activating stimulation [28]. IRF-1 has also been demonstrated to directly control TLR-9 mediated IFN- $\beta$ production in myeloid dendritic cells [43]. However, the role of IRF-1 in the inflammation involved in atherosclerosis and ACS has not been fully elucidated. The present study further supports the role of IRF-1 in the maturation and function of DCs in ACS patients.

As far as we known, DCs can be divided into three stages: DC precursors, immature DCs and mature DCs. It is reported that DC precursors and immature DCs cannot stimulate naïve T cells. Only mature DCs, which are characterized by the upregulation of adhesion molecules (CD11a, CD54, and CD58), co-stimulatory molecules (CD80, CD83 and CD86) and antigen-presenting molecules (MHC class I , II), could contact with naïve T cells and present antigens to those cells. Subsequently, naïve T cells differentiate into Th1- or Th2type cells through direct contact with mature DCs and the inflammatory cytokines (IL-12, TNF- $\alpha$ etc.) produced by mature DCs. Several studies have shown that DCs are activated and mature DCs are upregulated in patients with ACS $[35,44]$. In this study, we detected CD83, CD86 expression in DCs after inducing or silencing the expression of IRF-1. We demonstrated that both of these co-stimulatory molecules in DCs were significantly upregulated after the cells were transfected with pGC-FU-IRF-1. However, silencing the expression of IRF-1 may attenuate the maturation of DCs. Furthermore, we also found that the overexpression of IRF-1 in DCs significantly induced pro-inflammatory cytokine (IL-6, IL-12p70 and TNF- $\alpha$ ) production by DCs, whereas release of the anti-inflammatory cytokine IL-10 was reduced. In contrast, the inhibition of IRF-1 could decrease the secretion of pro-inflammatory cytokines 


\section{Cellular Physiology Cell Physiol Biochem 2015;36:599-610 \begin{tabular}{l|l|}
\hline DOI: 10.1159/000430123 & O 2015 S. Karger AG, Basel
\end{tabular} and Biochemistry Published online: May 18, 2015 \\ Guo et al.: IRF-1 Modulates the Function of DCs in ACS Patients}

and accelerate the secretion of the anti-inflammatory cytokine. These results indicate that IRF-1 may modulate the function of DCs by activating DC maturation as well as enhancing cytokine production and that IRF-1 plays a role in plaque instability and vulnerability towards rupture.

Many cells such as macrophages, monocytes or vascular smooth cells accumulate in the arterial intima and can engulf oxidatively modified lipids, particularly ox-LDL and differentiate into foam cells, which constitute a large component of atherosclerosis lesions $[45,46]$. Recent studies found that DCs also participate in cholesterol metabolism and may engulf ox-LDL and differentiate into foam cells. The presence of DC-derived foam cells has been identified using electron microscopy in human thoracic artery lesions [47]. We examined the immune effect of IRF-1 on the differentiation of DCs into foam cells. Our data clearly showed that the overexpression of IRF-1 could significantly increase ox-LDL uptake by DCs in vitro. In contrast, inhibiting the expression of IRF-1 decreased ox-LDL uptake by DCs. These results suggest that IRF-1 exerts a promotive effect on DC foam-cell formation. Three scavenger receptors, SRA, CD36 and LOX-1-1, which are expressed by macrophages and monocyte-derived DCs, are major receptors for oxLDL [48]. To further investigate whether SRA, CD36 and LOX-1-1 were required in the DC foam-cell formation, we examined the relative mRNA and protein expression levels of these scavenger receptors. The results confirmed that IRF-1 could significantly increase the expression of LOX-1 and activate the oxLDL uptake capacity of DCs, which could be related to the initiation and progression of ACS.

Thus, the exactly signaling mechanisms involved in the action of IRF- 1 in the pathogenesis of atherosclerosis and ACS remains undefined. In this study, we confirmed that the MAPK pathway (phosphorylation of JNK, p38 and ERK1/2) may participate in the signaling mechanism of the IRF-1 in the DCs of patients with ACS.

In summary, our study showed that the transcription factor IRF-1 may positively promote the plaque destabilization and the initiation of ACS via the activation of DC maturation and ox-LDL uptake. This newly discovered function of IRF-1 may help us to understand the mechanisms by which IRF-1 involved in the pathogenesis of atherosclerosis and ACS and may provide a novel target to manipulate the development of atherosclerosis and the onset of ACS.

\section{Acknowledgements}

This work was supported by Natural Science Foundation for Young Scientists of Shanxi Province, China (2013021035-2) to Min Guo.

\section{Disclosure Statement}

No conflict of interest.

\section{Reference}

1 Hansson GK, Hermansson A: The immune system in atherosclerosis. Nat Immunol;12:204-212.

2 Hansson GK, Libby P, Schonbeck U, Yan ZQ: Innate and adaptive immunity in the pathogenesis of atherosclerosis. Circ Res 2002;91:281-291.

3 Libby P, Ridker PM, Hansson GK: Progress and challenges in translating the biology of atherosclerosis. Nature 2011;473:317-325.

4 deGoma EM, Knowles JW, Angeli F, Budoff MJ, Rader DJ: The evolution and refinement of traditional risk factors for cardiovascular disease. Cardiol Rev 2012;20:118-129.

5 Lin J, Chang W, Dong J, Zhang F, Mohabeer N, Kushwaha KK, Wang L, Su Y, Fang H, Li D: Thymic stromal lymphopoietin over-expressed in human atherosclerosis: Potential role in th17 differentiation. Cell Physiol Biochem 2013;31:305-318. 


\section{Cellular Physiology Cell Physiol Biochem 2015;36:599-610 \begin{tabular}{l|l|l}
\hline DOI: 10.1159/000430123 & (C) 2015 S. Karger AG, Basel
\end{tabular} and Biochemistry Published online: May 18, 2015

6 Niessner A, Sato K, Chaikof EL, Colmegna I, Goronzy JJ, Weyand CM: Pathogen-sensing plasmacytoid dendritic cells stimulate cytotoxic t-cell function in the atherosclerotic plaque through interferon-alpha. Circulation 2006;114:2482-2489.

7 Daissormont IT, Christ A, Temmerman L, Sampedro Millares S, Seijkens T, Manca M, Rousch M, Poggi M, Boon L, van der Loos C, Daemen M, Lutgens E, Halvorsen B, Aukrust P, Janssen E, Biessen EA: Plasmacytoid dendritic cells protect against atherosclerosis by tuning t-cell proliferation and activity. Circ Res 2011;109:1387-1395.

8 Arlt O, Schwiebs A, Japtok L, Ruger K, Katzy E, Kleuser B, Radeke HH: Sphingosine-1-phosphate modulates dendritic cell function: Focus on non-migratory effects in vitro and in vivo. Cell Physiol Biochem 2014;34:27-44.

9 Jongstra-Bilen J, Haidari M, Zhu SN, Chen M, Guha D, Cybulsky MI: Low-grade chronic inflammation in regions of the normal mouse arterial intima predisposed to atherosclerosis. J Exp Med 2006;203:20732083.

10 Liu P, Yu YR, Spencer JA, Johnson AE, Vallanat CT, Fong AM, Patterson C, Patel DD: Cx3cr1 deficiency impairs dendritic cell accumulation in arterial intima and reduces atherosclerotic burden. Arterioscler Thromb Vasc Biol 2008;28:243-250.

11 Erbel C, Sato K, Meyer FB, Kopecky SL, Frye RL, Goronzy JJ, Weyand CM: Functional profile of activated dendritic cells in unstable atherosclerotic plaque. Basic Res Cardiol 2007;102:123-132.

12 Bobryshev YV, Taksir T, Lord RS, Freeman MW: Evidence that dendritic cells infiltrate atherosclerotic lesions in apolipoprotein e-deficient mice. Histol Histopathol 2001;16:801-808.

13 Ma-Krupa W, Jeon MS, Spoerl S, Tedder TF, Goronzy JJ, Weyand CM: Activation of arterial wall dendritic cells and breakdown of self-tolerance in giant cell arteritis. J Exp Med 2004;199:173-183.

14 Pryshchep 0, Ma-Krupa W, Younge BR, Goronzy JJ, Weyand CM: Vessel-specific toll-like receptor profiles in human medium and large arteries. Circulation 2008;118:1276-1284.

15 Niessner A, Weyand CM: Dendritic cells in atherosclerotic disease. Clin Immunol 2010;134:25-32.

16 Ranjit S, Dazhu L, Qiutang Z, Yibo F, Yushu L, Xiang W, Shen CL, Yuan T: Differentiation of dendritic cells in monocyte cultures isolated from patients with unstable angina. Int J Cardiol 2004;97:551-555.

17 Wang L, Li D, Yang K, Hu Y, Zeng Q: Toll-like receptor-4 and mitogen-activated protein kinase signal system are involved in activation of dendritic cells in patients with acute coronary syndrome. Immunology 2008;125:122-130.

18 Zenke M, Hieronymus T: Towards an understanding of the transcription factor network of dendritic cell development. Trends Immunol 2006;27:140-145.

19 Taniguchi T, Ogasawara K, Takaoka A, Tanaka N: Irf family of transcription factors as regulators of host defense. Annu Rev Immunol 2001;19:623-655.

20 Testa U, Stellacci E, Pelosi E, Sestili P, Venditti M, Orsatti R, Fragale A, Petrucci E, Pasquini L, Belardelli F, Gabriele L, Battistini A: Impaired myelopoiesis in mice devoid of interferon regulatory factor 1. Leukemia 2004;18:1864-1871.

21 Ogasawara K, Hida S, Azimi N, Tagaya Y, Sato T, Yokochi-Fukuda T, Waldmann TA, Taniguchi T, Taki S: Requirement for irf-1 in the microenvironment supporting development of natural killer cells. Nature 1998;391:700-703.

22 Matsuyama T, Kimura T, Kitagawa M, Pfeffer K, Kawakami T, Watanabe N, Kundig TM, Amakawa R, Kishihara K, Wakeham A, et al.: Targeted disruption of irf-1 or irf-2 results in abnormal type i ifn gene induction and aberrant lymphocyte development. Cell 1993;75:83-97.

23 Lohoff M, Ferrick D, Mittrucker HW, Duncan GS, Bischof S, Rollinghoff M, Mak TW: Interferon regulatory factor-1 is required for a t helper 1 immune response in vivo. Immunity 1997;6:681-689.

24 Tada Y, Ho A, Matsuyama T, Mak TW: Reduced incidence and severity of antigen-induced autoimmune diseases in mice lacking interferon regulatory factor-1. J Exp Med 1997;185:231-238.

25 Nakazawa T, Satoh J, Takahashi K, Sakata Y, Ikehata F, Takizawa Y, Bando SI, Housai T, Li Y, Chen C, Masuda T, Kure S, Kato I, Takasawa S, Taniguchi T, Okamoto H, Toyota T: Complete suppression of insulitis and diabetes in nod mice lacking interferon regulatory factor-1. J Autoimmun 2001;17:119-125.

26 Buch T, Uthoff-Hachenberg C, Waisman A: Protection from autoimmune brain inflammation in mice lacking ifn-regulatory factor-1 is associated with th2-type cytokines. Int Immunol 2003;15:855-859.

27 Guo M, Mao X, Ji Q, Lang M, Li S, Peng Y, Zhou W, Xiong B, Zeng Q: Inhibition of ifn regulatory factor-1 downregulate th1 cell function in patients with acute coronary syndrome. J Clin Immunol 2010;30:241-252. 


\section{Cellular Physiology Cell Physiol Biochem 2015;36:599-610 \begin{tabular}{l|l} 
DOI: 10.1159/000430123 & (C) 2015 S. Karger AG, Basel
\end{tabular} www.karger.com/cpb \\ Guo et al.: IRF-1 Modulates the Function of DCs in ACS Patients}

28 Gabriele L, Fragale A, Borghi P, Sestili P, Stellacci E, Venditti M, Schiavoni G, Sanchez M, Belardelli F, Battistini A: Irf-1 deficiency skews the differentiation of dendritic cells toward plasmacytoid and tolerogenic features. J Leukoc Biol 2006;80:1500-1511.

29 Schmid E, Xuan NT, Zahir N, Russo A, Yang W, Kuhl D, Faggio C, Shumilina E, Lang F: Serum- and glucocorticoid-inducible kinase 1 sensitive nf-kappab signaling in dendritic cells. Cell Physiol Biochem 2014;34:943-954.

30 Johnson GB, Brunn GJ, Samstein B, Platt JL: New insight into the pathogenesis of sepsis and the sepsis syndrome. Surgery 2005;137:393-395.

31 Pinsky MR: Dysregulation of the immune response in severe sepsis. Am J Med Sci 2004;328:220-229.

32 Hansson GK: Inflammation, atherosclerosis, and coronary artery disease. N Engl J Med 2005;352:16851695.

33 Wang B, Peng Y, Dong J, Lin J, Wu C, Su Y, Fang H, Wang L, Huang K, Li D: Human platelets express functional thymic stromal lymphopoietin receptors: A potential role in platelet activation in acute coronary syndrome. Cell Physiol Biochem 2013;32:1741-1750.

34 Fang H, Lin J, Wang L, Xie P, Wang X, Fu J, Ai W, Chen S, Chen F, Zhang F, Su Y, Li D: Kruppel-like factor 2 regulates dendritic cell activation in patients with acute coronary syndrome. Cell Physiol Biochem 2013;32:931-941.

35 Fukunaga T, Soejima H, Irie A, Fukushima R, Oe Y, Kawano H, Sumida H, Kaikita K, Sugiyama S, Nishimura Y, Ogawa H: High ratio of myeloid dendritic cells to plasmacytoid dendritic cells in blood of patients with acute coronary syndrome. Circ J 2009;73:1914-1919.

36 Tailor P, Tamura T, Ozato K: Irf family proteins and type i interferon induction in dendritic cells. Cell Res 2006;16:134-140.

37 Kung HC, Evensen O, Hong JR, Kuo CY, Tso CH, Ngou FH, Lu MW, Wu JL: Interferon regulatory factor-1 (irf1) is involved in the induction of phosphatidylserine receptor (psr) in response to dsrna virus infection and contributes to apoptotic cell clearance in chse-214 cell. Int J Mol Sci 2014;15:19281-19306.

38 Mboko WP, Mounce BC, Emmer J, Darrah E, Patel SB, Tarakanova VL: Interferon regulatory factor 1 restricts gammaherpesvirus replication in primary immune cells. J Virol 2014;88:6993-7004.

39 Wessely R, Hengst L, Jaschke B, Wegener F, Richter T, Lupetti R, Paschalidis M, Schomig A, Brandl R, Neumann FJ: A central role of interferon regulatory factor-1 for the limitation of neointimal hyperplasia. Hum Mol Genet 2003;12:177-187.

40 Tamura T, Ishihara M, Lamphier MS, Tanaka N, Oishi I, Aizawa S, Matsuyama T, Mak TW, Taki S, Taniguchi T: An irf-1-dependent pathway of DNA damage-induced apoptosis in mitogen-activated tymphocytes. Nature 1995;376:596-599.

41 Tanaka N, Ishihara M, Kitagawa M, Harada H, Kimura T, Matsuyama T, Lamphier MS, Aizawa S, Mak TW, Taniguchi T: Cellular commitment to oncogene-induced transformation or apoptosis is dependent on the transcription factor irf-1. Cell 1994;77:829-839.

42 Romeo G, Fiorucci G, Chiantore MV, Percario ZA, Vannucchi S, Affabris E: Irf-1 as a negative regulator of cell proliferation. J Interferon Cytokine Res 2002;22:39-47.

43 Schmitz F, Heit A, Guggemoos S, Krug A, Mages J, Schiemann M, Adler H, Drexler I, Haas T, Lang R, Wagner $\mathrm{H}$ : Interferon-regulatory-factor 1 controls toll-like receptor 9-mediated ifn-beta production in myeloid dendritic cells. Eur J Immunol 2007;37:315-327.

44 Kofler S, Sisic Z, Shvets N, Lohse P, Weis M: Expression of circulatory dendritic cells and regulatory t-cells in patients with different subsets of coronary artery disease. J Cardiovasc Pharmacol 2011;57:542-549.

45 Lusis AJ: Atherosclerosis. Nature 2000;407:233-241.

46 Weber C, Zernecke A, Libby P: The multifaceted contributions of leukocyte subsets to atherosclerosis: Lessons from mouse models. Nat Rev Immunol 2008;8:802-815.

47 Paulson KE, Zhu SN, Chen M, Nurmohamed S, Jongstra-Bilen J, Cybulsky MI: Resident intimal dendritic cells accumulate lipid and contribute to the initiation of atherosclerosis. Circ Res 2010;106:383-390.

48 Nickel T, Schmauss D, Hanssen H, Sicic Z, Krebs B, Jankl S, Summo C, Fraunberger P, Walli AK, Pfeiler S, Weis M: Oxldl uptake by dendritic cells induces upregulation of scavenger-receptors, maturation and differentiation. Atherosclerosis 2009;205:442-450. 